Note

\title{
Insoluble Dietary Fiber of Wheat Bran Increased Viscosity of Pig Whole Cecal Contents in Vitro
}

\author{
Takashi SAKATA, Masaaki SAITO \\ School of Science and Engineering, Ishinomaki Senshu University, 986-8580 Ishinomaki, Japan \\ (Received August 17, 2006)
}

\begin{abstract}
Summary Viscosity of whole pig cecal contents with $(2 \mathrm{~g} / 200 \mathrm{~mL})$ or without insoluble dietary fibers prepared from wheat bran of two particle sizes (WB fine $0.22 \pm 0.22 \mathrm{~mm}^{2}$, $n=1,723$; WB coarse $0.51 \pm 1.12 \mathrm{~mm}^{2}, n=1,457$, mean \pm SD) was measured using a rotary viscometer equipped with a vane spindle at the shear rates of $0.05,0.16,0.47,0.78$ and $1.56\left(\mathrm{~s}^{-1}\right)$ and at 0 and $2 \mathrm{~h}$. Such measurements were repeated twice each for five different donor pig groups. We were able to measure the coefficient of viscosity of such samples over a wide range of shear rate. Coefficient of viscosity depended on shear rate. Addition of insoluble dietary fiber increased the coefficient of viscosity. Insoluble dietary fiber from WB coarse had a significantly stronger effect than that from WB fine. The above results demonstrated that insoluble dietary fibers of wheat bran increase the viscosity of gut contents, and thereby potentially retard digestion and absorption.
\end{abstract}

Key Words insoluble dietary fiber, wheat bran, viscosity, digesta, vane spindle

We demonstrated that solid particles are mainly responsible for the viscosity of gut contents $(1,2)$, which is a major determinant of mixing and diffusion rates for enzymes, substrates and products. Thus, it is likely that the addition of solid food particles increases the viscosity of gut contents, and thereby reduces rates of digestion and absorption. If this was the case, then insoluble dietary fibers should have another nutritional function, which is to reduce rates of digestion and absorption of nutrients. Actually, the addition of cellulose particles reduced the absorption of glucose independent of adsorption or dilution, depending on the increase in the viscosity of gut contents in rats (3). However, this study used a refined basal diet without large particles leaving it unknown if the addition of insoluble dietary fibers of actual food origin increase the viscosity of gut contents with large food particles. Therefore, we tested the effect of insoluble dietary fiber prepared from wheat bran on the viscosity of pig cecal contents with large food particles in vitro. So far, the measurement of whole gut contents with large particles has been employing a tube-flow viscometer. However, this method was tedious and required a large sample. Therefore, the second objective of the present study was to measure the viscosity of whole gut contents including large particles using a rotary viscometer equipped with a vane spindle.

\section{Materials and Methods}

We used two types of commercial wheat bran with different particle sizes (WB fine $0.22 \pm 0.22 \mathrm{~mm}^{2}, n=$ 1,723; WB coarse $0.51 \pm 1.12 \mathrm{~mm}^{2}, n=1,457$, mean \pm

E-mail: sakata@isenshu-u.ac.jp
SD) and pooled pig cecal contents sampled from commercially slaughtered healthy meat pigs at Miyagi Meat Inspection Center, Tome, Japan. We adopted pig cecal contents as model mammalian gut contents, because pig cecal contents has basic flow behaviors in common with contents of other gut segments and of other mammalian and avian species (2).

We prepared insoluble dietary fiber from the two types of wheat bran according to AOAC method (4). The yield of insoluble dietary fiber fraction was 49.4 and $51.8(\mathrm{~g} / 100 \mathrm{~g})$ for WB fine and WB coarse, respectively. Each $2 \mathrm{~g}$ of insoluble fiber of one of these samples was suspended in $200 \mathrm{~mL}$ of fresh pig cecal contents and maintained at $37^{\circ} \mathrm{C}$ in a glass culture flask with a water jacket. We measured the coefficient of viscosity (shear stress/shear rate, $\mathrm{mPa} \cdot \mathrm{s}$ ) (1) of these samples and of pig cecal contents without wheat bran fiber (negative control) using a rotary viscometer (HBDV-E, Brookfield Engineering Laboratories Inc., Middleboro) equipped with a vane spindle (V-71, ibid.) (5) at the shear rates of $0.05,0.16,0.47,0.78,1.56\left(\mathrm{~s}^{-1}\right)$ and at 0 and $2 \mathrm{~h}$. Such measurements were repeated twice each for five different donor pig groups. Logarithmic means of coefficient of viscosity were compared with the Tukey HSD test after preliminary analysis of variance. The difference was considered significant when error probability was smaller than 0.05 .

\section{Results and Discussion}

We were able to measure coefficients of viscosity using the above stated setting. Shear stress of all samples depended on shear rate with positive yield stress, a non-Newtonian characteristic, agreeing with previous studies $(1,2)$. Coefficient of viscosity depended on donor 


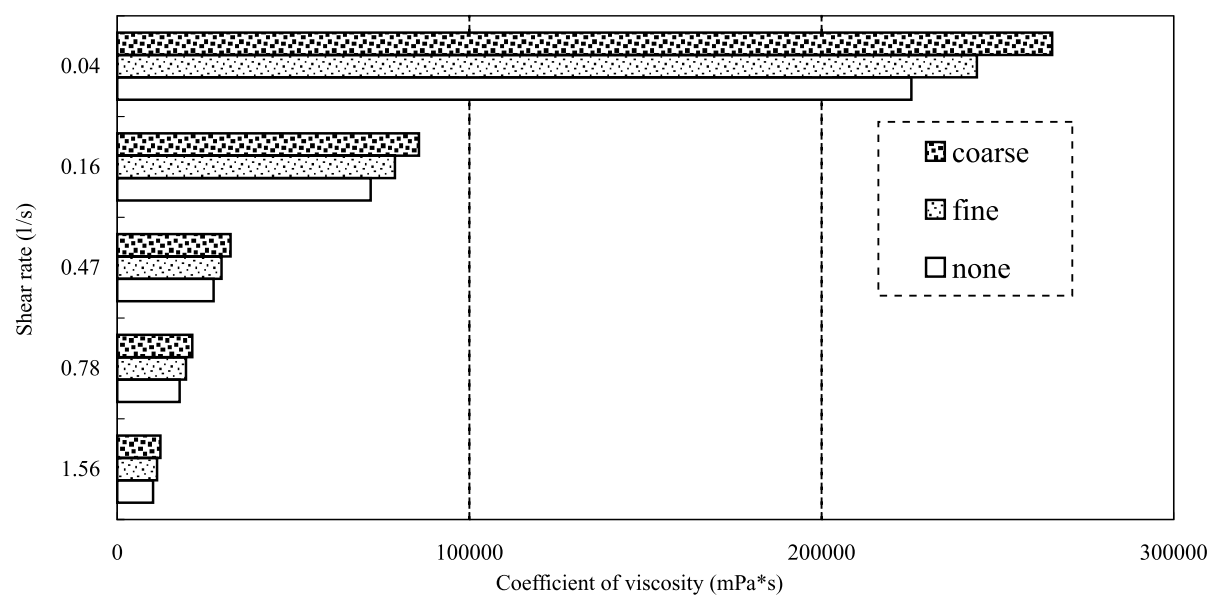

\begin{tabular}{|c|c|c|c|c|c|}
\hline Factor & $\mathrm{DF}$ & SS & F-value & Prob $>F$ & Comparison \\
\hline Donor group & 4 & 3.0901 & 103.9 & 0.0001 & $2=4=5>3>1$ \\
\hline Replicate & 1 & 0.0782 & 10.5 & 0.0014 & $1 \mathrm{st}>2 \mathrm{nd}$ \\
\hline Hour & 1 & & 35.5 & 0.0001 & $0 \mathrm{~h}>2 \mathrm{~h}$ \\
\hline Fiber type (F) & 2 & 0.0606 & 4.1 & 0.0188 & Coarse $>$ Fine $>$ None \\
\hline Shear rate (S) & 4 & 36.0234 & 1211.0 & 0.0001 & Negative \\
\hline $\mathrm{F} \times \mathrm{S}$ interaction & 8 & 0.0006 & 0.0 & 1.0000 & \\
\hline Error & 159 & 1.1825 & & & \\
\hline Total & 179 & 45.4680 & & & \\
\hline
\end{tabular}

Fig. 1. Means of coefficient of viscosity of pig cecal contents added with coarse, fine or no insoluble dietary fiber particles prepared from wheat bran with summary of analysis of variance.

pigs, replication and time of measurement, and shear rate (Fig. 1). Addition of particles significantly increased the coefficient of viscosity. Coarse particles had a significantly stronger effect than fine particles.

The above results demonstrated that solid food particles such as the insoluble fiber fraction of wheat bran are able to increase the viscosity of gut contents, and thereby potentially retard autoenzymatic digestion in the small intestine and alloenzymatic digestion in the large intestine. The increase in the viscosity by the addition of insoluble fiber ( $18 \%$ for coarse particle and $7 \%$ for fine particle) was comparable to that in a previous study (3) where the increase in the viscosity of gut contents reduced glycemic response independent of dilution or adsorption. This supports the idea of using less or hardly digestible solid foods such as whole grain cereals to depress the digestion and absorption of digestible starchy foods and thereby reduce the glycemic response, especially in those who have impaired glycemic response.

\section{Acknowledgments}

This study was partly supported by a research grant from Iijima Memorial Foundation for the Promotion of Food Science. We appreciate the kind help of meat inspection officers at Miyage Meat Inspection Center at the sampling of pig cecal contents.

\section{REFERENCES}

1) Takahashi T, Sakata T. 2002. Large particles increase viscosity and yield stress of pig cecal contents without changing basic viscoelastic properties. J Nutr 132: 1026-1030.

2) Takahashi T, Sakata T. 2005. Insoluble dietary fibers: The major modulator for the viscosity and flow behavior of digesta. FFI J 210: 944-951.

3) Takahashi T, Karita S, Ogawa N, Goto M. 2005. Crystalline cellulose reduces plasma glucose concentrations and stimulates water absorption by increasing the digesta viscosity in rats. J Nutr 135: 2405-2410.

4) AOAC Method 991.42. 1995. Insoluble dietary fiber in food and food products-Enzymatic-gravimetric method, phosphate buffer. In: Official Methods of Analysis, 16th ed. AOAC International, Gaithersburg, MD.

5) Brookfield Engineering Laboratories, Inc. 2006. SSV Vane Standard Spindle Set Assembly \& Operating Instructions Manual No. M02-290-A0803. Brookfield Engineering Laboratories, Inc, Middleboro, MA. 